Regular Paper

\title{
Shape Optimization for Magnet and Flux Barriers of IPMSM By Using Polygon Model Method with GP
}

\author{
Kota ISHIKAWA ${ }^{* 1}$, Wataru KITAGAWA ${ }^{* 1}$ and Takaharu TAKESHITA* ${ }^{* 1}$
}

\begin{abstract}
This paper presents the shape optimization of magnet and flux barriers in the interior permanent magnet synchronous motor (IPMSM). Authors use the polygon model method with genetic programming (GP) by the twodimensional finite element method (2D-FEM). In this method, the analysis model of IPMSM is regarded as a tree structure. This method optimizes a shape by operating tree structures with GP. The tree structure of this method is possible to express every shape. Therefore, this method can obtain an optimal shape without being bound by a stereotype. The purpose is the investigation of shape design of magnet and flux barriers to improve the electromagnetic characteristics. Additionally, authors take into consideration about complexity of shape and volume of magnet.
\end{abstract}

Keywords: finite element method, shape optimization, polygon model method, genetic programming, interior permanent magnet synchronous motor.

(Received: 24 July 2014, Revised: 28 April 2015, Revised: 29 May 2015, Revised: 1 July 2015)

\section{Introduction}

Recently, they are required high efficiency for electromagnetic machinery in environmental and energy problems. Therefore, this paper presents the shape optimization of rotor in the interior permanent magnet synchronous motor (IPMSM) which is adopted as the benchmark in The Institute of Electrical Engineers of Japan. IPMSM have been studied actively because it has a high degree of freedom of shape design [1-2]. Additionally, this motor can be used the magnet torque and reluctance torque. For these reasons, it has characteristics of high efficiency and high torque. Researchers use the finite element method (FEM) and optimization algorithm in order to optimize a shape of rotor in IPMSM [3-5]. However, these methods are often limited by using a size optimization method within a limited range and predetermined design parameters. Therefore, it has the disadvantage that depends on experiences and stereotypes of developer. The mainstream is the researches of topology and shape optimization by using such as a level-set and on-off method. They have attracted attention as the method that can be used in engineering recently [6-8].

In this paper, authors present one of approaches about these optimizations. It is the polygon model method by operating tree structures. The tree structure of rotor in IPMSM is shown in Fig. 1. The analysis model like this structure is composed of some objects that have the data of some vertexes and materials. This proposed method is the structural optimization by operating these tree structures. The tree structure is

\footnotetext{
Correspondence: W. KITAGAWA, Graduate School of Engineering, Nagoya Institute of Technology, Gokiso, Showa, Nagoya, Aichi 466-8555, Japan email: kitagawa.wataru@nitech.ac.jp

${ }^{* 1}$ Nagoya Institute of Technology
}

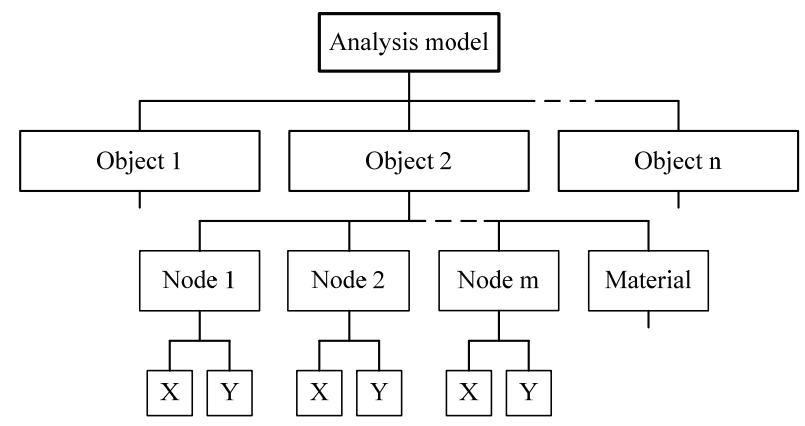

Fig. 1. Tree structure of analysis model that is composed of objects.

possible to express every form and shape. Moreover, it is able to use an optimization algorithm together. This method has a more versatile optimization because it is possible to obtain the improvement of characteristics and simple form and shape by setting of objective functions and constraint conditions.

The optimization algorithm of this paper is genetic programming (GP) [9]. GP is the extended method of genetic algorithm (GA) in order to adapt it for use the tree structure. The research of GP is popular in recent years, and it is possible to easily implement by a tree structure representation. The verification model is the IPMSM of 3 phases 4 poles 24 slots, and the search region is its rotor. The purpose is the investigation of shape design of magnet and flux barriers to improve the electromagnetic characteristics as the average steady torque and torque ripple. Additionally, authors take into consideration about complexity of shape and volume of magnet.

\section{Optimization method}

\subsection{Polygon model method}

The region of magnet and flux barrier in IPMSM is regarded as a certain object. This object is a polygon 
that is composed of some vertexes. The shape is optimized by operating these objects and their vertexes. A flux barrier is composed of some vertexes, and a magnet is composed of some vertexes and material information such as magnetization and its direction. The analysis model is composed of these objects as shown in Fig. 1. In a conventional method, the size optimization is based on the predetermined design parameters. However, the polygon model method is possible to search a shape within the entire region. This method has a high degree of freedom of shape design by optimization of tree structures because it is possible to express every shape. In this method, the expression of holes in an object is not taken into consideration. It is not the topology optimization, but the shape optimization. However, it is capable of the extension to topology optimization by applying this method.

\subsection{Genetic Programming (GP)}

GP is one of the metaheuristic algorithms in order to search an approximate solution. It is the extension of structural genotype about GA. In this method, an analysis model is regarded as a genotype tree structure as shown in Fig. 1. The optimal shape is searched by repeating a genetic operation such as the evaluation, selection, crossover, and mutation. The flowchart of proposed method is shown in Fig. 2.

First of all, the proposed method generates analysis models of the number of population randomly. The generation of initial population is necessary to specify the values of number of population, objects, nodes, and search radius. It uses the mersenne twister (MT) to generate random numbers. It uses master-slave method

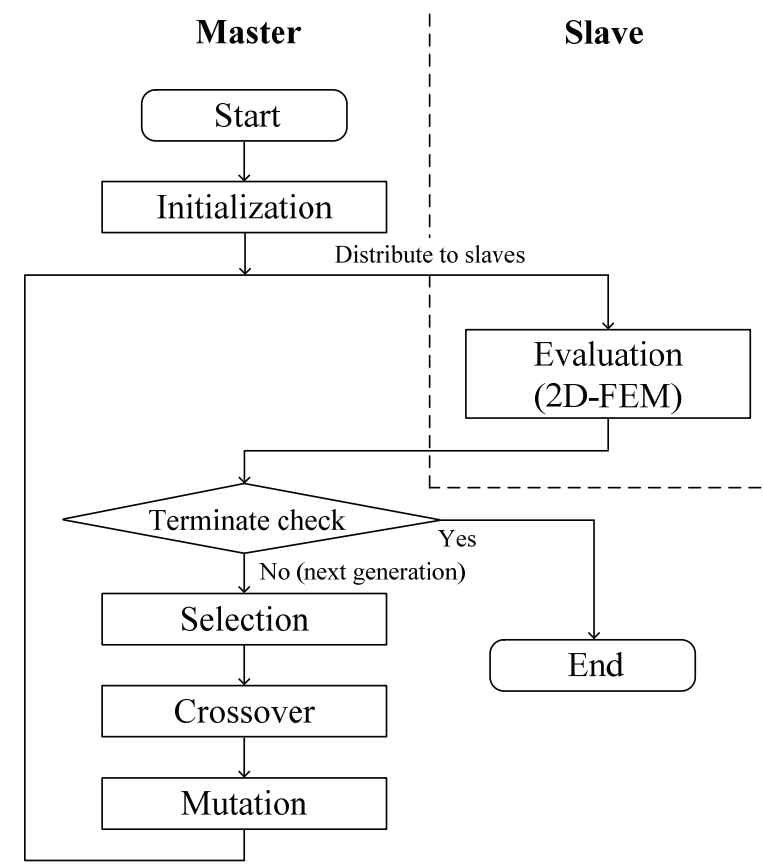

Fig. 2. Flowchart of proposed method by using GP.

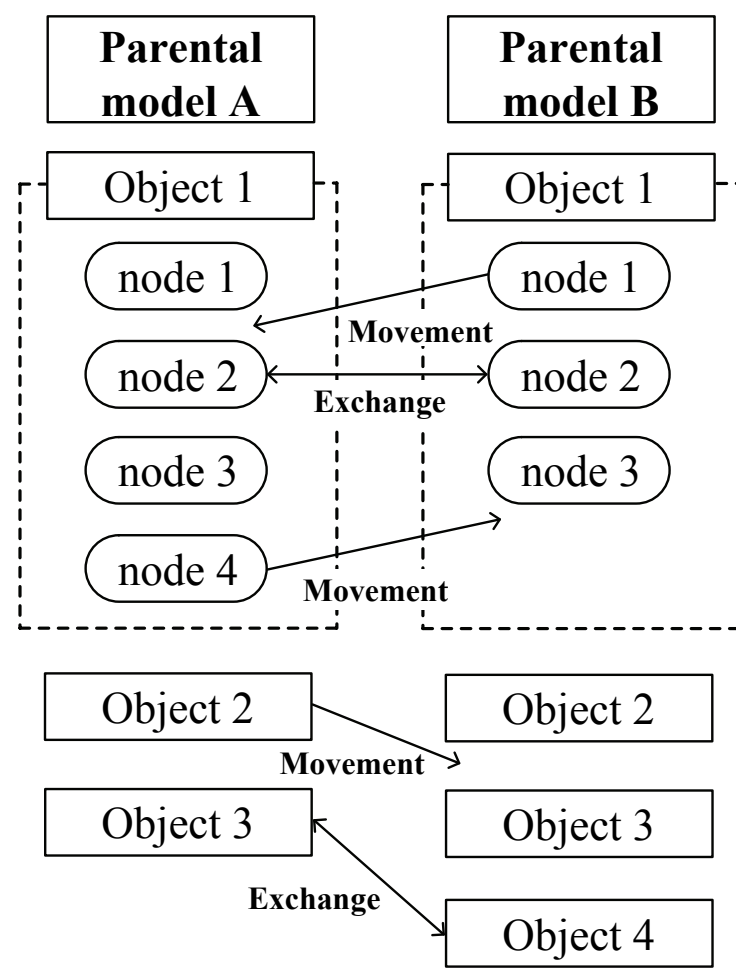

Fig. 3. Example of crossover by exchange and movement of nodes and objects.

in the parallel processing. The analysis models distribute to slaves, and they analyze the electromagnetic characteristics such as the steady torque by using 2DFEM. The automatic mesh generation is the bubble mesh method [10]. This method is used after the shape is determined.

The description of genetic operation is shown below. The proposed method uses evaluation, selection, crossover, and mutation for genetic operation. In the evaluation, it calculates a fitness value by the predetermined fitness functions of characteristics as the steady torque. In the selection, individuals of high fitness value are likely to be selected. It selects the number of population for the next generation. An example of crossover is shown in Fig. 3. In the crossover, it selects two parental models from the population of analysis models. The parental models cross each other by the crossover probability. The proposed method has two crossover methods. These are crossover of objects and nodes. The positions of crossover for nodes and objects in the parental models are selected randomly. Moreover, it performs the exchange and movement of values. Apart from the crossover probability of parents, it is necessary to set the crossover probabilities of nodes and objects. The total number of candidates for crossover of object and node is the number of the smaller model of those. Moreover, the number of candidates is determined by the crossover probability of object and node. They are divided into the method of exchange and movement. Additionally, they are divided into the method to send 
and receive in the movement by a probability of 0.5 . It is possible to give the child models without losing the genetic information of shape in the parental model by crossing only a part of object and node. The reason is because it has the order of nodes as well as the value of nodes. In the mutation, the operations are the addition, delete, and movement of objects. Similarly, nodes are operated by the addition, delete, and movement. In the operation of magnet, values of coordinates and rectangle are changed. These operations are performed by the mutation probability and each probability of these operations. The proposed method returns to the parallel processing after these genetic operations. These processing repeat until the termination condition is satisfied. As a result, the proposed method can search an optimal shape.

\subsection{Model check}

The generation of initial population and genetic operation are possibility that it generates an unanalyzable or wrong model. For example, it is the model having a self-intersection. It is necessary to prevent the generation for these models in the initial generation and genetic operation. Additionally, the models having the same nodes of an object and the overlap of region of objects are also prevented in this paper. The problem is resolved by checking the model when each item of initial generation and genetic operation. Its operation is repeated until acceptable model is generated. Therefore, the models can be generated without impossible models.

\subsection{Parallel processing}

It is necessary to evaluate many analysis models in the optimization. The computing cost is spent enormous. By the parallel processing, it is able to analyze the problem of large scale by a single PC. In this paper, analysis models are calculated by 4 PCs. It is possible to evaluate the individuals by the total $16 \mathrm{CPU}$ cores because each PC has $4 \mathrm{CPU}$ cores. GP is necessary to perform the global processing every generation. It is not possible to evaluate individuals about next generation until CPUs are ended all of the present evaluations. In this method, a computing time per individual is not long by using 2D-FEM. It analyzes an individual that has not been analyzed yet in order after an analysis by CPU has been completed. This reason is because they have time

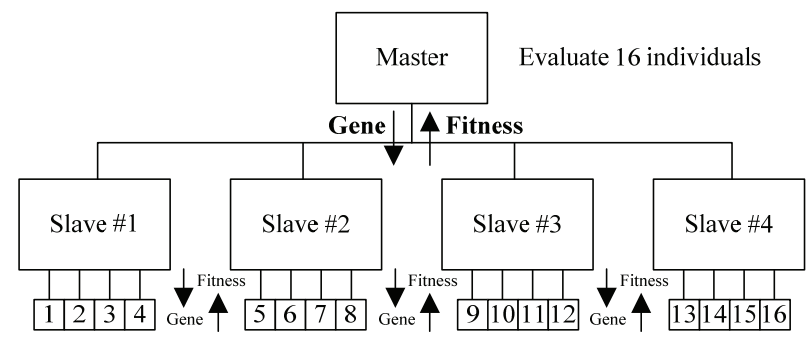

Fig. 4. Parallel processing for GP by master-slave method. difference for each individual. In the parallel calculation method, Authors use the master-slave method as shown in Fig. 4. In the master side, it performs the genetic operation and control of entire analysis. On the other hand, in the slave side, it calculates the fitness value by using 2D-FEM. It uses the text files with information like a fitness value between master and slave by using TCP/IP in order to exchange genetic information.

\section{Analysis Condition}

\subsection{Analysis model}

The mesh model of analysis model is shown in Fig. 5. The analysis model is the IPMSM which is adopted as the benchmark model in IEE of Japan [11]. This is the IPMSM of 3 phases 4 poles 24 slots. The analysis range is $0-90$ degrees from the cycle of its model. The counterclockwise is plus for the direction of rotation. The currents of each phase are expressed by the following equations.

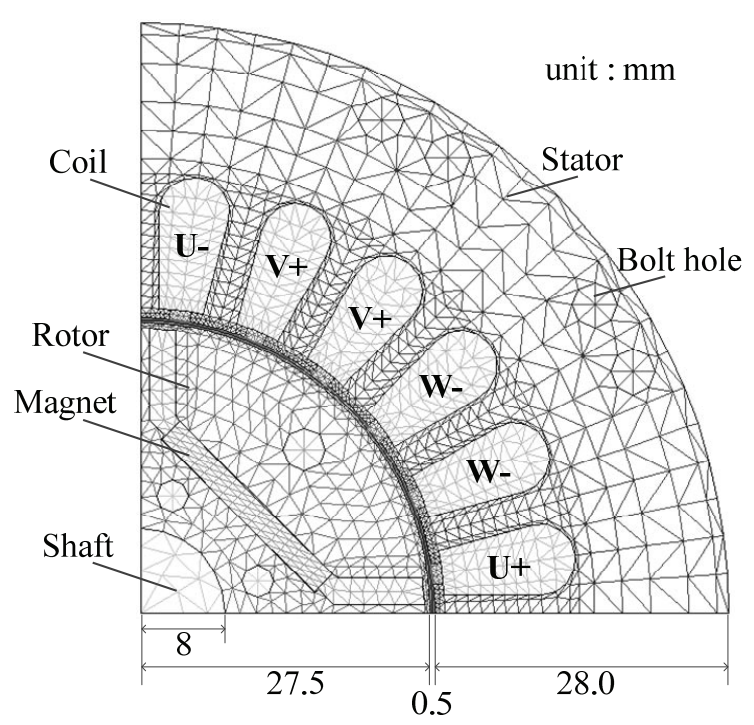

Fig. 5. Initial model and its specification.

Table 1 Specification of initial model.

\begin{tabular}{c|c}
\hline Material (Stator Core) & M-36 (JIS: 50A350) \\
\hline Material (Rotor Core) & M-36 (JIS: 50A350) \\
\hline Material (Shaft) & s45c \\
\hline Magnetization [T] & 1.25 \\
\hline Direction of magnetization & Parallel \\
\hline Turn / phase & 140 (4 coils in series) \\
\hline Current density [AT $\left./ \mathrm{m}^{2}\right]$ & $2.107 \times 10^{6}$ \\
\hline Laminated core [mm] & 60 \\
\hline Rated current Irms [A] & 3.0 \\
\hline Frequency of current [Hz] & 50 \\
\hline Speed [min $\left.{ }^{-1}\right]$ & 1500 \\
\hline Number of elements & 1478 \\
\hline Number of nodes & 2868 \\
\hline
\end{tabular}

CPU: Intel Core i 7 of $2.93 \mathrm{GHz} / \mathrm{MEM}: 8 \mathrm{~GB}$ 


$$
\begin{aligned}
& I_{U}=\sqrt{2} I_{r m s} \sin (\omega t+\beta) \\
& I_{V}=\sqrt{2} I_{r m s} \sin \left(\omega t-\frac{2}{3} \pi+\beta\right) \\
& I_{W}=\sqrt{2} I_{r m s} \sin \left(\omega t+\frac{2}{3} \pi+\beta\right)
\end{aligned}
$$

where $I_{r m s}$ is the effective value of current. $\omega$ is the angular frequency, $\beta$ is the current phase. The cycle of steady torque is 30 degrees in mechanical angle from the number of poles and shape geometry. Therefore, the number of analysis steps of steady torque is 31 . The specifications of the model are shown in Table 1 . The material of stator and rotor core is M-36. This is an electromagnetic steel sheet. The material of shaft is $\mathrm{s} 45 \mathrm{c}$. This is a carbon steel for machine structural use. The magnetization is $1.25 \mathrm{~T}$, and it is a neodymium magnet. This model is the initial model. The optimization of this method is started without magnet and flux barriers of initial model.

\subsection{Evaluation method}

GP is necessary to express the fitness value of each individual because it decides the superior individuals for next generation in the selection. In this paper, it calculates the average steady torque and its ripple by using 2D-FEM. These value is used the calculation of fitness value. The calculation of fitness value is expressed by the following equations.

$$
\begin{aligned}
& f=T_{a v e} \cdot w_{1}+T_{r p} \cdot w_{2}+C \cdot w_{3}-M \cdot w_{4} \\
& T_{\text {ave }}=\frac{T_{\text {ave_opt }}}{T_{\text {ave_ini }}} \\
& T_{r p}=\frac{T_{r p \_i n i}}{T_{r p \_i n i}+T_{r p \_o p t}} \\
& C=\frac{C_{\text {ini }}}{C_{\text {opt }}} \\
& C_{o p t, i n i}=\frac{l^{2}}{S} \\
& M= \begin{cases}0 & \left(M_{o p t}<M_{i n i}\right) \\
\frac{M_{o p t}}{M_{i n i}}-1 & \left(M_{o p t}>M_{i n i}\right)\end{cases}
\end{aligned}
$$

where $T_{a v e}$ is the average steady torque, $T_{r p}$ is its ripple, $C$ is the worst complexity of all object, and $M$ is the area of magnet. Additionally, the opt of subscript is the value of optimized model, and the ini of subscript is that of initial model. $l$ is the circumference of object and $S$ is the area of object. It calculates $T_{a v e}, T_{r p}, C$, and $M$ of the value by above functions. It multiplies these values by each weighting factor. $f$ is the fitness value. It is the sum of these values. The average steady torque is the value by the current phase angle that steady torque is maximized. The fitness value is set to increase by increasing the average steady torque, decreasing the ripple and complexity of object. In addition, its value is set to decrease when the area of magnet is larger than that of initial model. The complexity of object plays a filtering role of object.

\subsection{Shape condition}

In this paper, the method optimizes a magnet and flux barriers. This simultaneous optimization is necessary to express a tree structure in order to use this method. Shapes must be possible to use in engineering. In this paper, the magnet and flux barriers are imposed restrictions in model check. This method treats the magnet as a rectangle. The magnet has 3 parameters. These are coordinates and values of rectangle. The magnet faces the stator side in the position and direction of 45 degrees. The flux barriers add the edge of coordinates about magnet to the first of flux barriers and the first and second of its nodes. The search region of flux barriers is a half of rotor as shown in Fig. 6. It generates flux barriers so as to be symmetrical.

\subsection{Parameters}

In this method, it is necessary to decide the GP parameters, and the range of parameters in the model creation. The GP parameters are shown in Table 2, that of crossover is shown in Table 3, and that of mutation is shown in Table 4. The weighting factor of $w_{1}$ is $10, w_{2}$ is $3, w_{3}$ is 1 , and $w_{4}$ is 1 . The initial model is used as an air-conditioner. It is important to improve the average torque and torque ripple. The population size of GP parameter is 1000 in Table 2. It is necessary to prepare

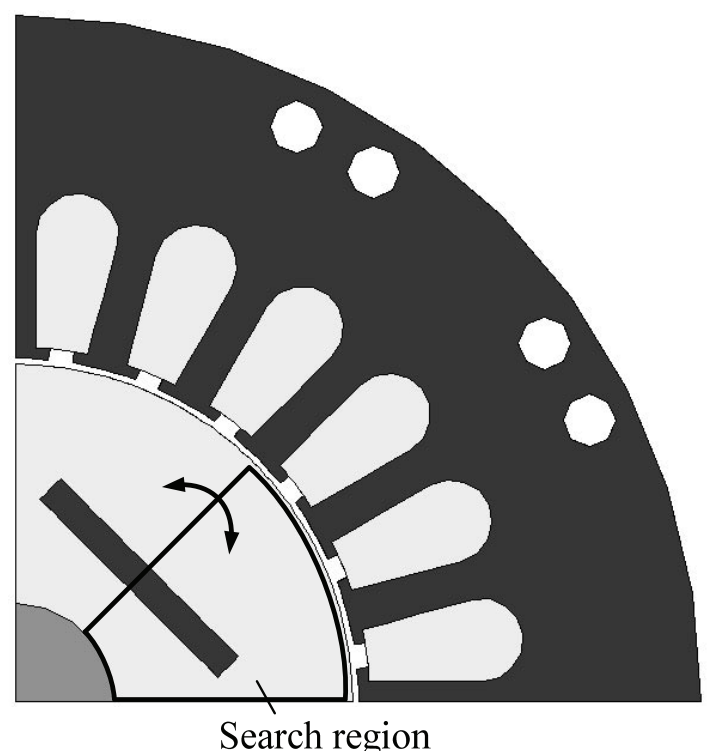

Fig. 6. Search region of flux barriers in IPMSM. 
Table 2 Specification of GP parameters.

\begin{tabular}{c|c}
\hline Population size & 1000 \\
\hline Selection method & $\begin{array}{c}\text { Tournament } \\
\text { with Elite }\end{array}$ \\
\hline Tournament size & 2 \\
\hline Elite size & 1 \\
\hline Crossover probability & 0.80 \\
\hline Mutation probability & 0.05 \\
\hline $\begin{array}{c}\text { Termination condition by } \\
\text { number of continuous generation }\end{array}$ & 30 \\
\hline
\end{tabular}

Table 3 Specification of crossover parameters.

\begin{tabular}{c|c}
\hline Crossover probability of object & 0.20 \\
\hline $\begin{array}{c}\text { Crossover probability of } \\
\text { node and material information }\end{array}$ & 0.20 \\
\hline (Method) Exchange & 0.50 \\
\hline (Method) Movement & 0.50 \\
\hline
\end{tabular}

Table 4 Specification of mutation parameters.

\begin{tabular}{c|c}
\hline (Target) Object & 0.20 \\
\hline (Method) Addition & 0.40 \\
\hline (Method) Delete & 0.30 \\
\hline (Method) Movement & 0.30 \\
\hline (Target) Node & 0.80 \\
\hline (Method) Addition & 0.40 \\
\hline (Method) Delete & 0.30 \\
\hline (Method) Movement & 0.30 \\
\hline (Target) Coordinates of magnet & 0.05 \\
\hline (Target) Rectangle of magnet & 0.15 \\
\hline
\end{tabular}

Table 5 Specification of parameter range.

\begin{tabular}{c|c}
\hline Initial number of objects & $1-3$ \\
\hline Initial number of nodes & $3-10$ \\
\hline Search radius [mm] & $8.0-27.0$ \\
\hline
\end{tabular}

much size as possible because this method by the GP deals with the huge number of solutions. The selection method is tournament and elite method. This method finishes its program after the termination condition is satisfied by the number of continuous generation. Table 3 shows the crossover probability of object and node after the decision of crossover for parental models. Additionally, it shows the probability of crossover method as the exchange and movement. As shown in Table 4, it selects the target and method. The target is an object or node, and the method is addition, delete, or movement.

The parameter range of model creation is shown in Table 5. This method is necessary to set the parameters of generation of initial population and mutation. The initial number of nodes must be at least 3 in order to have a region of object. The search radius is up to 27.0 $\mathrm{mm}$ because it has the necessary room of $0.5 \mathrm{~mm}$ from a structural problem. The minimum value is $8.0 \mathrm{~mm}$ because the shaft is not included in the search radius.

\section{Optimization result}

\subsection{Evaluation process}

The evaluation process by proposed method is shown in Fig. 7. The end of generation is 179 and fitness value is 17.46 because the termination condition

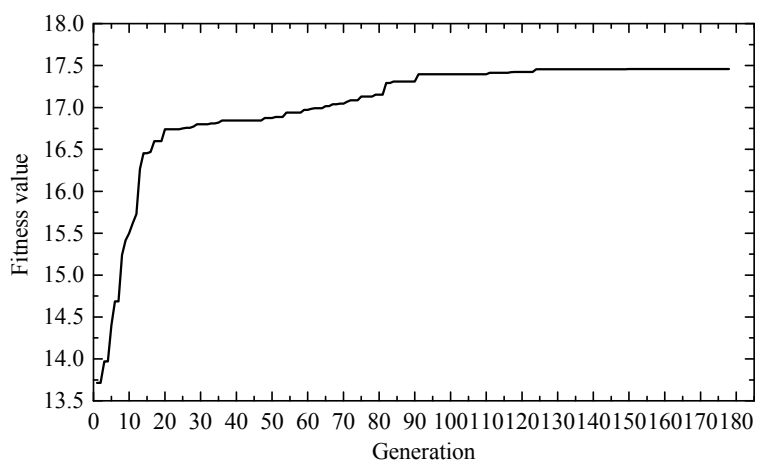

Fig. 7. Evaluation process by proposed method.

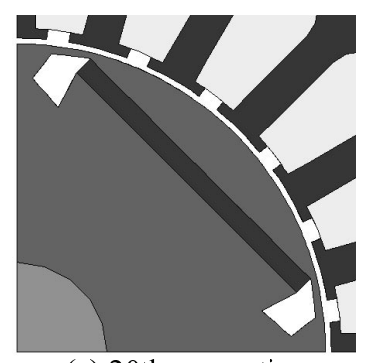

(a) 20th generation

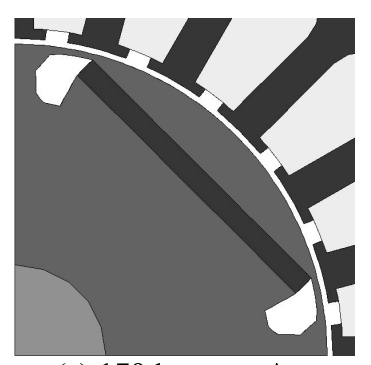

(c) 179th generation

Fig. 8. Optimized shape of magnet and flux barriers.

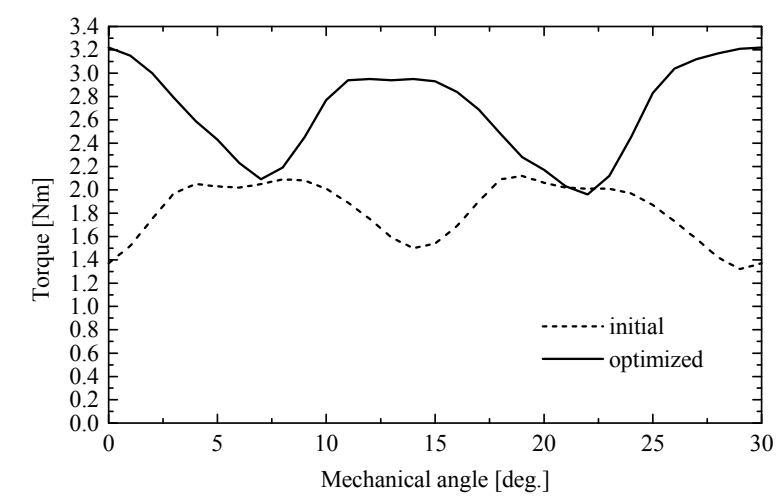

Fig. 9. Steady torque waveform of optimized shape. (179th generation) 
Table 6 Summary of characteristics

(20th generation).

\begin{tabular}{c|c|c}
\hline & initial & optimized \\
\hline $\begin{array}{c}\text { Average steady } \\
\text { torque [Nm] }\end{array}$ & 1.833 & $\begin{array}{c}2.579 \\
(40.7 \% \uparrow)\end{array}$ \\
\hline $\begin{array}{c}\text { Ripple of } \\
\text { steady torque }\end{array}$ & 0.434 & $\begin{array}{c}0.515 \\
(18.7 \% \uparrow)\end{array}$ \\
\hline $\begin{array}{c}\text { Complexity of } \\
\text { flux barrier }\end{array}$ & 24.14 & $\begin{array}{c}18.56 \\
(23.1 \% \downarrow)\end{array}$ \\
\hline Area of magnet $\left[\mathrm{mm}^{2}\right]$ & 51.95 & $\begin{array}{c}47.89 \\
(7.8 \% \downarrow)\end{array}$ \\
\hline Fitness value & 12.50 & $\begin{array}{c}16.74 \\
(33.9 \% \uparrow)\end{array}$ \\
\hline
\end{tabular}

Table 7 Summary of characteristics.

(90th generation)

\begin{tabular}{c|c|c}
\hline & initial & optimized \\
\hline $\begin{array}{c}\text { Average steady } \\
\text { torque [Nm] }\end{array}$ & 1.833 & $\begin{array}{c}2.651 \\
(44.6 \% \uparrow)\end{array}$ \\
\hline $\begin{array}{c}\text { Ripple of } \\
\text { steady torque }\end{array}$ & 0.434 & $\begin{array}{c}0.477 \\
(9.9 \% \uparrow)\end{array}$ \\
\hline $\begin{array}{c}\text { Complexity of } \\
\text { flux barrier }\end{array}$ & 24.14 & $\begin{array}{c}16.99 \\
(29.6 \% \downarrow)\end{array}$ \\
\hline Area of magnet $\left[\mathrm{mm}^{2}\right]$ & 51.95 & $\begin{array}{c}51.24 \\
(1.4 \% \downarrow)\end{array}$ \\
\hline Fitness value & 12.50 & $\begin{array}{c}17.31 \\
(38.5 \% \uparrow)\end{array}$ \\
\hline
\end{tabular}

Table 8 Summary of characteristics.

(179th generation)

\begin{tabular}{c|c|c}
\hline $\begin{array}{c}\text { Average steady } \\
\text { torque [Nm] }\end{array}$ & initial & optimized \\
\hline $\begin{array}{c}\text { Ripple of } \\
\text { steady torque }\end{array}$ & 0.434 & $\begin{array}{c}2.667 \\
(45.5 \% \uparrow)\end{array}$ \\
\hline $\begin{array}{c}\text { Complexity of } \\
\text { flux barrier }\end{array}$ & 24.14 & $\begin{array}{c}16.473 \\
(9.0 \% \uparrow)\end{array}$ \\
\hline Area of magnet $\left[\mathrm{mm}^{2}\right]$ & 51.95 & $\begin{array}{c}52.01 \\
(0.1 \% \uparrow)\end{array}$ \\
\hline Fitness value & 12.50 & $\begin{array}{c}17.46 \\
(39.7 \% \uparrow)\end{array}$ \\
\hline
\end{tabular}

by number of continuous generation is 30 . The total computing time is 170.2 hours. It is realistic time. The fitness value of initial model is 12.50 . The end value of evaluation is higher than the value of initial model.

\subsection{Optimized shape and characteristics}

The optimized shapes are shown in Fig. 8, and the steady torque waveform is shown in Fig. 9. Moreover, the summaries of its characteristics are shown in Table 6-8. The magnet appears in the surface side of rotor, and the flux barriers appear on both sides of magnet from Fig. 8. Additionally, the shapes of flux barrier are not complicated. The areas of magnet are much the same.

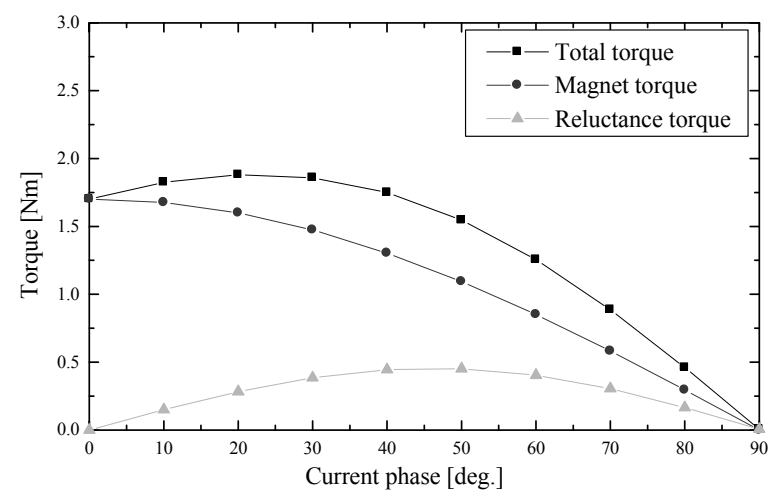

Fig. 10. Magnet and reluctance torque. (initial)

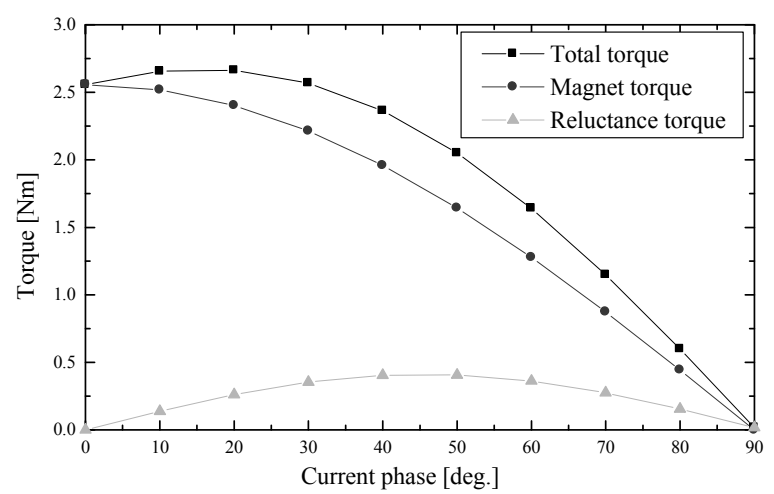

Fig. 11. Magnet and reluctance torque. (optimized)

The average steady torque of optimized shape is increased $45.5 \%$ compared with initial shape. However, the ripple of optimized shape is increased $9.0 \%$ compared with initial shape. This is due to the distribution of weighting factors. It is necessary to change the value of weighting factors, or to implement multi-objective optimization.

The ratios of magnet torque and reluctance torque are shown in Fig. 10 and Fig. 11. In these figures, the magnet torque of optimized model is higher than that of initial model. Therefore, the average torque of optimized model is increased because the magnet torque is increased.

\section{Conclusion}

This paper presents a new method of shape optimization by the polygon model method with GP. This method is high degree of freedom of shape design because a tree structure is possible to express every shape. The purpose is simultaneous optimization of shape of magnet and flux barriers in order to improve the electromagnetic characteristics. Moreover, the complexity of object and volume of magnet are taken into consideration. As a result of this method, the average steady torque of optimized shape was improved 
$45.5 \%$ compared with initial shape. Simultaneously, it was possible to obtain simple shape of flux barriers.

In the future works, authors will investigate the parameter identification and filtering of shape design. These parameters are the polygon model method and GP parameters. It is necessary to search the best parameters. In the engineering, the shapes and characteristics of magnet and flux barriers are taken into consideration. Therefore, authors will consider the filtering method and problem of multi-objective optimization.

\section{References}

[1] G. W. Cho, S. H. Woo, S. H. Ji, K. B. Jang, and G. T. Kim, "Optimization of rotor shape for constant torque characteristic of IPM motor," ICEMS, pp. 1-4, 2011.

[2] P. Alotto, M. Barcaro, N. Bianchi, and M. Guarnieri, "Optimization of Interior PM Motors With Machaon Rotor Flux Barriers," IEEE Trans. Magn., Vol.47, No.5, pp.958-961, 2011.

[3] P. D. Barba and M. E. Mognaschi, "Industrial design with multiple criteria: Shape optimization of a permanentmagnet generator," IEEE Trans. Magn., Vol.45, No.3, pp.1482-1485, 2009.

[4] Y. Kawase, T. Yamaguchi, M. Mizuno, T. Nakano, and K Tanaka, "Characteristics Analysis of IPM Motor Driven by Voltage Source Inverter with Parallel Computing," Proceedings of the 19th International Conference on Electrical Machines ICEM2010, 2010.

[5] Y. Kawase, T. Yamaguchi, M. Yoshida and K. Hirata, "3D Finite Element Analysis of Rotary Oscillatory Actuator Using A New Auto Mesh Coupling Method," Digests of The 9th Biennial IEEE Conference on Electromagnetic Field Computation, 401, 2000.

[6] J. S. Choi, K. Izui, S. Nishiwaki, A. kawamoto, and T. Nomura, "Topology Optimization of the Stator for Minimizing Cogging Torque of IPM Motors," IEEE Trans. Magn., Vol.47, No.10, pp.3024-3027, 2011.

[7] S. Park, S. Min, S. Yamasaki, S. Nishiwaki, and J. Yoo, "Magnetic Actuator Design Using Level Set Based Topology Optimization," IEEE Trans. Magn., Vol.44, No.11, pp.4037-4040, 2008.

[8] N. Takahashi, T. Yamada, and D. Miyagi, "Examination of Optimal Design of IPM Motor Using ON/OFF Method," IEEE Trans. Magn., Vol.46, No.8, pp.3149-3152, 2010.

[9] Y. Suttasupa, S. Rungraungsilp, S. Pinyopan, P. Wungchusunti, and P. Chongstitvatana, "A comparative study of linear encoding in Genetic Programming," ICT and Knowledge Engineering (2011)., pp.13-17, 2012.

[10] T. Yokoyama, V. Cingoski, K. Kaneda, and H. Yamashita, "3-D automatic mesh generation for FEA using dynamic bubble system," IEEE Trans. Magn., Vol.35, No.3, pp.1318-1321, 1999.

[11] Investigating R\&D Committee on Practical Performance Evaluation Techniques of Rotating Machines by Electromagnetic Field Analysis, "Practical Performance Evaluation Techniques of Rotating Machines by Electromagnetic Field Analysis," 2012. (in Japanese) 